\author{
Maestría en Economía \\ Facultad de Ciencias Económicas \\ Universidad Nacional de La Plata
}

TESIS DE MAESTRIA

ALUMNO

Andrés Loza

TITULO

Análisis de la Competitividad del Mercado de Leche en la Argentina

\title{
DIRECTOR
}

Germán Coloma

FECHA DE DEFENSA

$6 / 22 / 2001$ 


\section{ANÁLISIS DE LA COMPETITIVIDAD DEL MERCADO PRIMARIO DE LECHE EN ARGENTINA}

\section{Resumen}

El mercado mayorista de leche en Argentina muestra una estructura apropiada para que las empresas tengan poder de mercado y se apropien de parte de la renta que correspondería a los productores rurales en un entorno competitivo. La presente investigación utiliza las modernas técnicas de la Nueva Organización Industrial Empírica, para resolver si las empresas mayoristas elaboradoras de leche ejercen su poder de mercado sobre el productor o tambero. Tanto las pruebas paramétricas y no paramétricas utilizadas coinciden en afirmar que existe cierto grado de poder monopsónico en ese mercado. También se muestra que el grado de ejercicio de poder de mercado es máximo en los meses de pico de producción.

\footnotetext{
${ }^{1}$ Tesis de la Maestría en Economía de la UNLP dirigida por el Dr. Germán Coloma
} 


\section{Introducción}

El mercado primario de leche presenta ciertas características específicas que lo diferencian del resto de las materias primas alimenticias. A partir de esas particularidades se presume que podría haber lugar para que las empresas elaboradoras de leche ejerzan poder de mercado sobre los productores rurales. Esto crearía una mala asignación de los recursos, con pérdidas económicas para los productores, derivadas de fallas en el mercado por la presencia de un comportamiento anticompetitivo, demandando la acción de una política económica en ese mercado.

La leche es un producto altamente perecedero y solamente a través de la elaboración de productos puede encontrarse una manera de almacenamiento que, salvo para la leche en polvo, es irreversible por la imposibilidad de volver a reconstruir la leche. La poca durabilidad de la leche sin procesar sumada a los altos costos de transporte pueden limitar la competitividad a nivel nacional. Veríamos, entonces, operando a unas pocas empresas grandes, acaparando el mercado, y a una gran cantidad de tambos atomizados. Esta estructura sería apropiada para que las grandes empresas puedan ejercer algún grado de poder monopsónico en el mercado local, donde el precio se fija libremente entre los productores y las empresas mayoristas.

El objetivo de la presente investigación es acumular evidencia empírica suficiente para determinar si en el período bajo análisis hubo ejercicio de poder de mercado por parte de las empresas mayoristas en la determinación del precio primario de la leche en Argentina.

El período sobre el que se desarrollará la investigación será el comprendido entre los años1992 y 1997. Algunos hechos que diferencian éste período de los anteriores son los siguientes:

1) Algunos documentos empíricos describen cierto cambio estructural en la técnica de producción utilizada por los tamberos. El sistema de producción hasta la década de 1990 era básicamente pastoril, con acentuadas diferencias entre los pisos de producción en el invierno y los picos en el verano. El trabajo de Parellada y Schilder 
(1999), llega a la conclusión de que a partir del año 1992, comienza a disminuir el peso del componente estacional y aumenta el cíclico, debido a la incorporación de reservas forrajeras en el esquema alimenticio de la vaca. De esa manera, el mejor aprovechamiento de los pastos permitió que disminuyeran las variaciones estacionales de la producción.

2) El ritmo de crecimiento se ha acelerado como consecuencia de la mayor productividad del tambo.

3) "En los comienzos de la década del 90 aparecen tres hechos clave: el Plan de Convertibilidad en la Argentina, la apertura de los mercados del este europeo y la finalización de la Ronda Uruguay del GATT. Estas situaciones determinaron cambios fundamentales dentro del esquema de decisiones empresariales tanto a nivel del tambo como a nivel industrial" (Parellada y Schilder, op. cit.).

El desarrollo de la investigación consistirá en aplicar las distintas formas de análisis que pueden extraerse de la teoría de la organización industrial, adaptándolas al caso del mercado mayorista lácteo argentino. En el capítulo II se comenzará con un análisis apreciativo de los datos que determinen algún indicio de poder de mercado, completando con el uso de indicadores de estructura en la línea del paradigma estructura-conducta-desempeño. En los capítulos III y IV se utilizan las técnicas más modernas de la "Nueva Organización Industrial Empírica", que compite con el paradigma anterior. En el capítulo III hacemos uso de pruebas no paramétricas y, en el capítulo IV, de las estimaciones econométricas de un modelo. En el capítulo V se expone la conclusión.

\section{Análisis descriptivo}

Como primera aproximación, realicé un análisis de las series sin ningún tipo de modelo económico. El gráfico 1 muestra la serie de producción nacional de leche. Sin pretender hacer un análisis econométrico exhaustivo de las series, quiero resaltar que la producción muestra una tendencia ascendente con amplias fluctuaciones, debido a que tiene un importante componente estacional. 


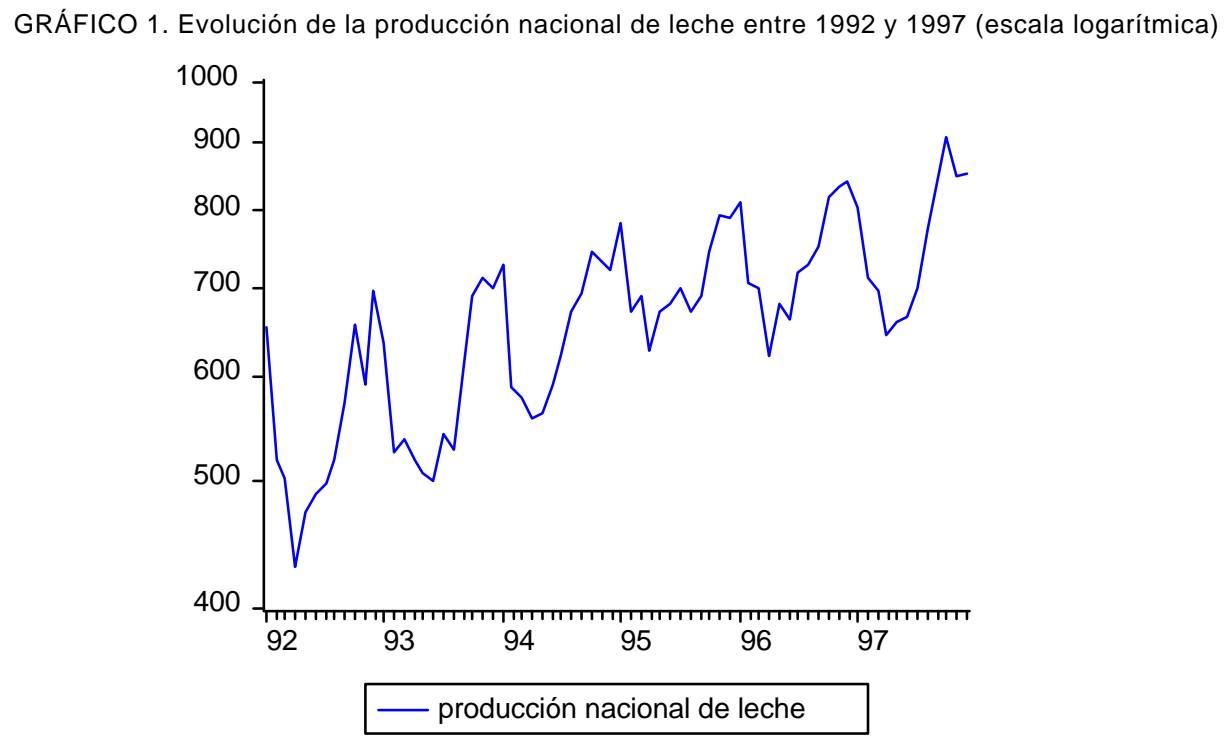

Fuente: Secretaría de Agricultura, Ganadería, Pesca y Alimentación.

Más vinculado con el problema central del trabajo es preguntarse qué ocurre con los precios relativos entre el insumo y el producto elaborado. El gráfico 2 muestra las series de precios pagados al productor y de la leche pasteurizada en el mercado minorista en pesos corrientes.

GRAFICO 2. Evolución de los precios pagados al productor y de la leche pasteurizada al por menor entre 1992 y 1997.

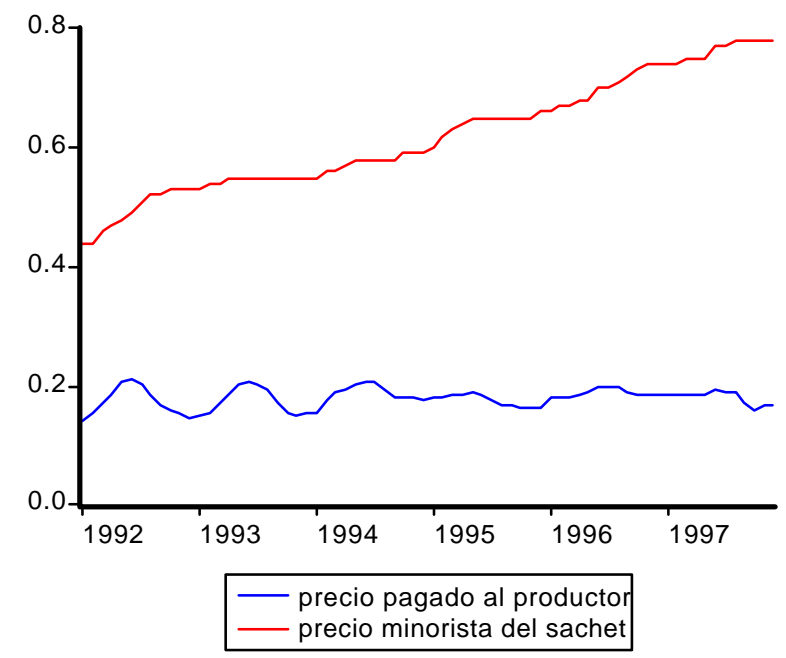

Fuente: Secretaría de Agricultura, Ganadería, Pesca y Alimentación y INDEC.

Puede observarse que hay una divergencia entre estas dos series. Mientras los precios que recibe el productor oscilan en algo menos que $\$ 0,20$, el precio final se dispara de manera constante durante el período analizado. Habría de esta manera algún 
indicio de ejercicio de poder de mercado. Aunque la leche con destino a leches fluidas representa aproximadamente un $25 \%$ de la producción total, se observaron otras series de precios al consumidor disponibles, y todas mostraban una tendencia positiva.

Ahora es el momento de hacer uso de la teoría de la organización industrial en el marco del paradigma estructura-conducta-desempeño. Como medidas de estructura se calcularon índices de Herfaindal y de concentración para las empresas compradoras de leche cruda para el año $1996^{2}$.

Para el total del país el índice de Herfaindal es cercano al 6\%, indicando un irrisorio grado de concentración. Pero si se analiza más de cerca como se estructura el mercado en cuestión, nos encontramos con grandes empresas que dominan dentro de una región. De esta manera un análisis por regiones o cuencas lecheras daría una mejor caracterización de la estructura subyacente. El trabajo de SAPyA (1996) sobre las cuencas lecheras argentinas, tiene información sobre las principales empresas que actúan en cada cuenca. El cuadro 1 sintetiza la información de SAPyA (1996).

Como puede apreciarse, Sancor es la líder en Santa Fe, donde compiten 197 empresas, mientras que La Serenísima es la principal en Buenos Aires, donde compiten 276 según SAPyA (1996). Además, estas dos principales empresas no aparecen como grandes competidoras en ninguna cuenca en común.

El $\mathrm{C}^{3}$ alcanza el valor de 41,6 , pero de vuelta caen las consideraciones para el índice anterior. De todas maneras, el índice de concentración resulta más apropiado para el caso en estudio, ya que la estructura nos muestra que hay pocas empresas dominantes

\footnotetext{
${ }^{2}$ La información se extrajo de Lamisovky(1998).

${ }^{3}$ El índice de concentración para las cuatro principales empresas.
} 


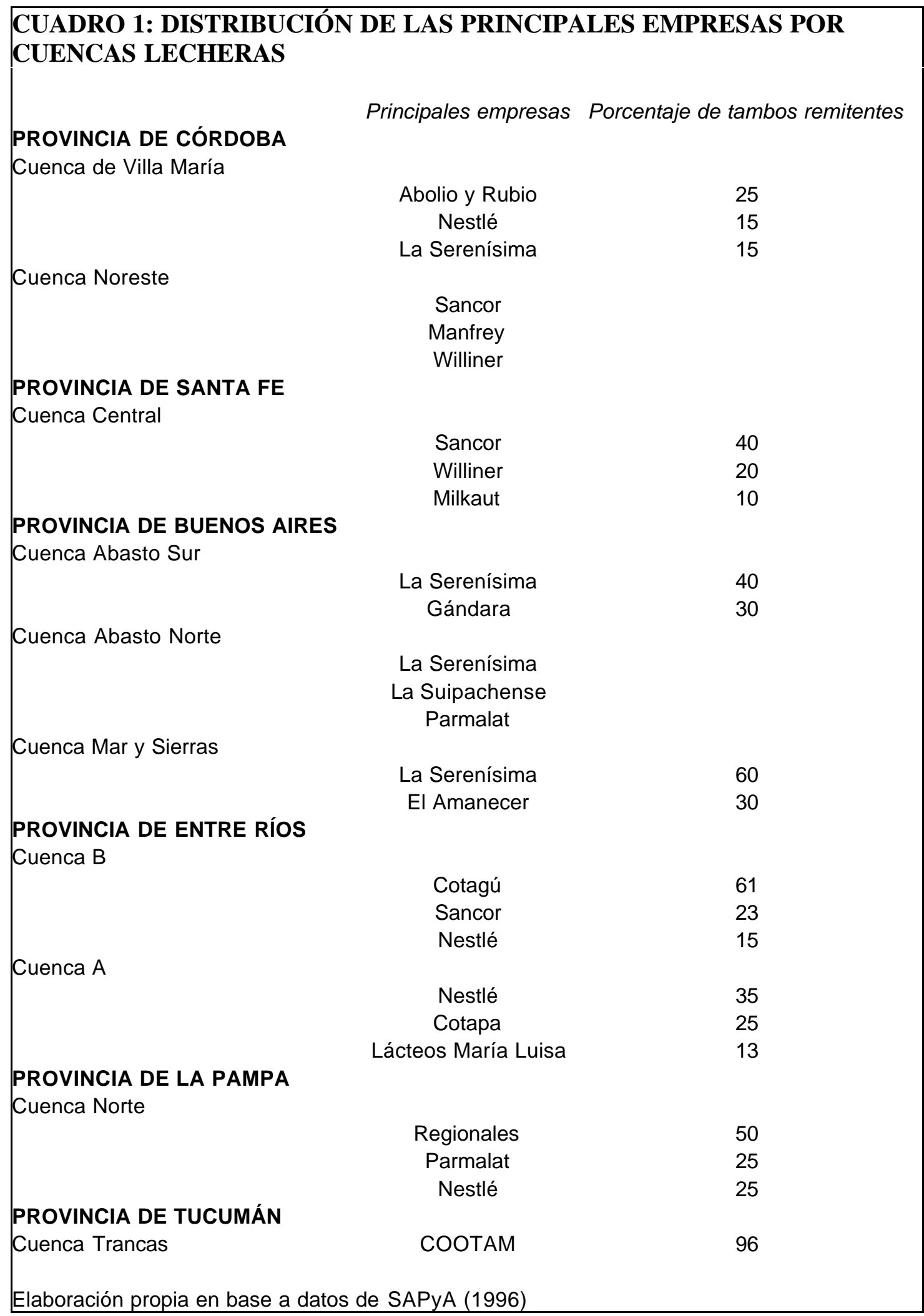


y muchas empresas con una participación extremadamente pequeña compitiendo por un producto homogéneo. ${ }^{4}$

Lo expuesto en este capítulo sirve para caracterizar una estructura lejana a la que supone el modelo de competencia. Sin llegar al grado extremo de monopsonio, los productores podrían estar expuestos a cierto grado intermedio de poder de mercado. Las series de precios y cantidades ya presentan algún indicio en esa dirección.

\section{Estimaciones no paramétricas.}

Como segundo paso en el análisis de los datos de precios y cantidades, se utilizará una serie de pruebas no paramétricas. El espíritu de los tests no paramétricos está en intentar contrastar hipótesis mediante el uso crudo de los datos y sin hacer supuestos ad hoc sobre ninguna forma funcional, evitando así los errores de especificación incorrecta. Además, el uso de estas técnicas no paramétricas requiere de menos variables, ya que no es necesario conocer la oferta y la demanda.

Para el desarrollo de este capítulo se adaptarán los trabajos de Varian (1984, 1990, 1996) para el caso de monopsonio y se simplificará el test de ejercicio de poder de mercado para un monopsonio de Love and Shumway(1994), derivado también de la bibliografía de Varian. Estas pruebas intentarán aportar evidencia a favor o en contra del ejercicio de poder de mercado de las empresas lácteas hacia los productores. Como resultado complementario se estimará algún parámetro que luego pueda compararse con las posteriores pruebas paramétricas.

Los datos disponibles no están discriminados por empresa, obligando a trabajar con el supuesto analítico de que en el mercado actúa una sola empresa, y los resultados deberían interpretarse como para una empresa representativa de la industria. Cada resultado rechazará alguna de las posibilidades siguientes, para cada uno de los tests:

Caso A: la empresa actúa como si maximizara en competencia perfecta.

\footnotetext{
${ }^{4}$ Ver Martín (1993), pp 167-169.
} 
Caso B: la empresa actúa como maximizadora ejerciendo poder de mercado monopsónico.

Caso C: la empresa no maximiza beneficios.

El primer problema que hay que resolver es si se puede rechazar el "caso C" 5 . La importancia radica en que tanto el test de Love and Shumway como las posteriores estimaciones paramétricas se derivan de la hipótesis de una conducta que persigue la maximización del beneficio. Las dos primeras pruebas servirán para ver si puede rechazarse el "caso C" y la tercera, si no es rechazado el supuesto de maximización, procederá a rechazar el "caso A" o el "caso B".

Los datos a utilizar corresponden a series mensuales para el año 1996 y 1997, que es sólo un subconjunto de los que se usarán en las estimaciones paramétricas. La ventaja de trabajar con un período no muy amplio es que se puede prescindir del progreso tecnológico dentro del análisis. Esto simplifica no solo la técnica de las estimaciones, sino que además permite trabajar con todos los datos conjuntamente. Por ejemplo, si existiera cambio tecnológico del tipo no-regresivo, las violaciones al supuesto de maximización podrían deberse a que es posible producir la misma cantidad con menos nivel de insumos o más cantidad con el mismo nivel de insumos comparando contra un período anterior a la introducción de las nuevas tecnologías. De esa manera, no deberían compararse los datos de un período con los posteriores a este, ya que las técnicas del futuro no estarían disponibles. En este trabajo evitamos esta problemática, suponiendo que el estado de las artes está dado durante el período analizado.

Prueba 1: Violación del axioma débil de la maximización del beneficio en el caso de competencia. La primer pregunta es si las observaciones superan el modelo de competencia. En este caso, si la empresa maximiza beneficios debería cumplirse lo que Varian(1984) llamó el axioma débil de la maximización de beneficios:

\footnotetext{
${ }^{5}$ Lo que implicaría que la empresa aproxima su comportamiento al de algún modelo que puede resolverse bajo el supuesto de maximización, sea este de competencia, oligopsonio o monopsonio.
} 
(1) $p^{t} y^{t} \geq p^{t} y^{s} \quad(t, s=1, \ldots, n)$,

donde $\boldsymbol{p}$ es el vector de precios e $\boldsymbol{y}$ el vector de producciones netas.

Si en alguna observación se viola la relación anterior, podemos argumentar que no se maximizan beneficios. Pero como sugiere Varian (1990), este criterio es demasiado estricto; habría que preguntarse más bien en términos económicos que lejos se está de la maximización de beneficios. Como un indicador razonable, Varian ofrece el siguiente:

$$
a^{t s}=p^{t}\left(y^{s}-y^{t}\right) / p^{t} y^{t}=p^{t} y^{s} / p^{t} y^{t}-1
$$

que es el porcentaje adicional a los precios $t$, que podría haberse obtenido en los beneficios usando otra de las técnicas disponibles.

Como criterio para este trabajo se considerará que las observaciones rechazan el comportamiento maximizador si conjuntamente superan el 5\%.

El promedio final para todos los meses es de $4,20^{6}$, que se encuentra dentro del nivel de significación elegido para no rechazar el "caso A". El siguiente paso será trabajar suponiendo que el modelo relevante es el de monopsonio. Si nuevamente se rechazara el "caso C", podría concluirse que es apropiado suponer que la empresa actúa como maximizadora. Este supuesto es imprescindible para la realización del último test.

Prueba 2: Violación de la hipótesis de maximización en un monopsonio. Si la empresa es monopsonista, enfrenta la curva de oferta del productor de materia prima. La condición de maximización del beneficio deberá incorporar la curva de oferta subjetiva que la empresa utiliza para su toma de decisiones. Adaptando el análisis de Varian (1984) en competencia imperfecta para el caso de monopsonio, se sigue que la hipótesis de maximización del beneficio implica:

\footnotetext{
${ }^{6}$ Los resultados de este capítulo se obtuvieron con MATHEMATICA ${ }^{\circledR}$ 3.0.
} 
$p^{t} y^{t}-r^{t} x^{t} \geq p^{t} y^{s}-r^{t s} x^{s}$

donde $\mathrm{r}^{\mathrm{ts}}$ es el precio que el empresario espera que prevalezca en la situación $t$ si produce $\boldsymbol{y}^{s}$ (demanda $\boldsymbol{x}^{s}$ ).

En el mismo artículo, Varian sugiere incorporar algunas restricciones. En este trabajo adaptamos dos para el caso de monopsonio y las utilizamos conjuntamente. La primera de ellas es la siguiente:

(4) $\left(\mathrm{r}^{\mathrm{t}}-\mathrm{r}^{\mathrm{ts}}\right)\left(\mathrm{x}^{\mathrm{t}}-\mathrm{x}^{\mathrm{s}}\right) \leq 0$,

exigiendo que la curva de oferta sea de pendiente positiva.

La segunda es que la oferta subjetiva coincida con la observación, es decir, $\mathrm{r}^{\mathrm{ts}}=\mathrm{r}^{\mathrm{s}}$. Combinando las dos restricciones se obtiene la utilizada en el trabajo:

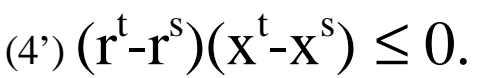

El indicador, para aquellas observaciones que hallan superado la restricción, es el siguiente:

$$
\begin{aligned}
b^{t s} & =\left\{p^{t}\left(y^{s}-y^{t}\right)-r^{s} x^{s}+r^{t} x^{t}\right\} /\left\{p^{t} y^{t}-r^{t} x^{t}\right\} \\
& =\left(p^{t} y^{s}-r^{s} x^{s}\right) /\left(p^{t} y^{t}-r^{t} x^{t}\right)-1
\end{aligned}
$$

El promedio general del índice es de 2,58, también por debajo del nivel de significación elegido. La maximización de beneficios se considera un supuesto razonable a la vista de los resultados. 
Todavía no se ha evaluado la hipótesis de ejercicio de poder de mercado. Dado que la prueba está basada en la hipótesis de maximización del beneficio, era conveniente que los datos superaran una primera batería de tests.

Prueba 3: Análisis sobre el poder de mercado. La prueba siguiente surge intuitivamente de Varian (1984). Love and Shumway(1994) desarrollan un test donde evalúan ejercicio de poder de mercado permitiendo la existencia de progreso técnico del tipo neutral en el sentido de Hicks. En este trabajo se simplificará el test de Love and Shumway, suponiendo que no se produce progreso tecnológico durante el período de análisis. El objetivo de la prueba es determinar que especificación de mercado resulta más apropiada: si la de competencia o la de monopsonio.

Reordenando la ecuación (3) y reemplazando $\mathrm{r}^{\mathrm{ts}}=\mathrm{r}^{\mathrm{s}}$,

(6) $p^{t}\left(y^{t}-y^{s}\right)-r^{t} x^{t}+r^{s} x^{s} \geq 0$.

Sumando y restando $\mathrm{r}^{\mathrm{t}} \mathrm{x}^{\mathrm{s}}$, obtengo:

(6) $p^{t}\left(y^{t}-y^{s}\right)-r^{t}\left(x^{t}-x^{s}\right)-x^{s}\left(r^{t}-r^{s}\right) \geq 0$.

Si se actuara en competencia perfecta el último término de la ecuación (6’) sería cero, ya que la firma no tendría poder para alterar los precios variando la cantidad demandada.

La idea es imponer un parámetro sobre el último término que si toma el valor cero es porque los datos se racionalizan en competencia perfecta y, si es positivo, cuanto más elevado mayor sea el grado de imperfección. Luego ese parámetro debería ser compatible con el índice de Lerner $^{7}$ para la medición de poder de mercado. A

\footnotetext{
${ }^{7}$ Este índice fue desarrollado por Lerner (1934) para el caso de monopolio como el cociente de la diferencia entre el precio y el costo marginal, sobre el precio.
} 
continuación se derivará a partir de las condiciones de maximización, una adaptación para el caso de monopsonio.

El problema de la maximización del beneficio para el monopsonista de la industria láctea se plantea de la siguiente forma:

$$
\begin{aligned}
& \max _{x_{1}, \ldots, x_{n}} \pi=\sum_{i=1}^{n} p_{i} y_{i}\left(x_{i}\right)-r(X) X \\
& X=\sum_{i=1}^{n} x_{i},
\end{aligned}
$$

donde $\boldsymbol{p}_{\boldsymbol{i}}$ es el precio del producto lácteo $\boldsymbol{i}$ comercializado en un mercado final competitivo $^{8}, \boldsymbol{y}_{\boldsymbol{i}}$ es la cantidad producida de $\boldsymbol{i}, \boldsymbol{x}_{\boldsymbol{i}}$ es la cantidad de leche destinada para elaborar el producto $\boldsymbol{i}$ y $\boldsymbol{n}$ el número de los diferentes productos lácteos que se elaboran. Los demás insumos que entran en la elaboración se dejan de lado en esta prueba como en las anteriores.

La condición de primer orden es la siguiente:

$$
p_{i} \frac{\partial y_{i}}{\partial x_{i}}-\frac{\partial r}{\partial X} X-r=0 \quad i=1, \ldots, n
$$

Sumando las condiciones de primer orden se obtiene:

$$
\sum_{i=1}^{n} p_{i} \frac{\partial y_{i}}{\partial x_{i}}-n \frac{\partial r}{\partial X} X-n r=0
$$

\footnotetext{
${ }^{8}$ Se considera por simplicidad y para no perder el objetivo de la tesis, que estos venden sus productos en competencia perfecta directamente a los consumidores. Es decir, se está obviando la comercialización al consolidar al mayorista con el minorista.
} 
Reordenando y dividiendo ambos miembros por $\boldsymbol{r}$ y $\boldsymbol{n}$ se obtiene la igualdad siguiente:

(7) $\frac{1}{\eta}=\frac{\partial r}{\partial X} \frac{X}{r}=\frac{1 / n \sum_{i=1}^{n} p_{i} \frac{\partial y_{i}}{\partial x_{i}}-r}{r}=\beta$.

Esta igualdad nos dice que en equilibrio, la inversa de la elasticidad-precio de la oferta del insumo, $\mathbf{1} / \mathbf{\eta}$, deberá igualar al valor de la producción de añadir una unidad más del insumo menos su costo en términos del precio. Es lo mismo que decir que iguala el beneficio neto de utilizar una unidad adicional del insumo en términos de lo que se paga por este. Al término de la derecha, $\boldsymbol{\beta}$, lo denominaremos "índice de Lerner", y será el indicador de la medida del porcentaje que sobre el precio es capaz de obtener el empresario, indicando su capacidad para deprimir el precio que paga al productor respecto del valor de la productividad marginal.

En términos discretos la elasticidad-precio de la oferta podría aproximarse por:

$$
\frac{\left(x^{t}-x^{s}\right)}{\left(r^{t}-r^{s}\right)} \frac{r^{t}}{x^{s}} \cong \eta^{t s}
$$

Defino el parámetro $\boldsymbol{m}^{t s}$ como:

$m^{t s}=\frac{r^{t}}{\eta^{t s}}$

Reemplazando $\boldsymbol{m}^{t s}$ en la ecuación (6’) tengo

(8) $p^{t}\left(y^{t}-y^{s}\right)-r^{t}\left(x^{t}-x^{s}\right)-m^{t s}\left(x^{t}-x^{s}\right) \geq 0$, 
que es otra alternativa para escribir la condición de maximización de beneficios. Si asumo la conducta maximizadora, puedo buscar el mínimo valor de los $\boldsymbol{m}^{t s}$ que consiguen racionalizarla. El mínimo valor de $\operatorname{los} \boldsymbol{m}^{t s}$ se resuelve igualando a cero la restricción y despejando. Es decir,

$$
m^{t s}=p^{t} \frac{\left(y^{t}-y^{s}\right)}{\left(x^{t}-x^{s}\right)}-r^{t} \quad \forall t \neq s=1,2, \ldots, T .
$$

El valor de los $\boldsymbol{m}^{t s}$ para cada par de observaciones permitirá estimar el correspondiente índice de Lerner dividiendo cada $\boldsymbol{m}^{t s}$ por $\boldsymbol{r}^{t}$.

La estimación del índice de Lerner se realizará para cada par de datos que superen la restricción (4'). En caso contrario, se estarían incluyendo cambios que no son consecuencia del ejercicio del poder de mercado. Como señalan Love and Shumway(1994), los desplazamientos de la curva de oferta del insumo que no son suficientemente compensados por desplazamientos de la curva de demanda correspondiente, podrían conducir a variaciones en la dirección opuesta para precios y cantidades, cambio que no puede ser atribuido al poder de mercado.

El índice de Lerner estimado es 2,44 como promedio total de todos los indicadores que superan la restricción (4'). Estos son 148 de las 552 posibles combinaciones. Eliminando 4 valores extremos ("outliers") cae a 1,45. Love and Shumway desalientan el uso del test como medida cardinal; en este trabajo se lo utilizará como medida comparativa de los resultados paramétricos. De todos modos, lo importante es ver en cuanto el índice se aparta de cero, dejando espacio a que efectivamente hubo ejercicio de poder de mercado y que la hipótesis de que el modelo correcto es el de competencia perfecta no sería una especificación adecuada.

Este test ha sido experimentado usando el método de Montecarlo en el trabajo de Noelke y Raper (1998). La conclusión a la que llegan es que detecta el poder de 
mercado monopsónico y en una magnitud que puede ser considerada económicamente significativa. De todos modos hay que tener en cuenta que los resultados son aceptables bajo la restricción de que el modelo especificado (monopsonio) es el correcto, es decir, es acertada la dirección en la que se evalúa el poder de mercado.

El trabajo de Lamisovsky(1998) sobre el mercado lácteo minorista en Argentina, concluye que no hay evidencia de ejercicio de poder monopólico; esta conclusión apoya la técnica del presente trabajo ${ }^{9}$.

Como conclusión de las estimaciones no paramétricas puede sostenerse la hipótesis de maximización del beneficio que, en una estructura de mercado monopsónico, implica el ejercicio de poder de mercado tal cual se planteó en el "caso B”. Más aún, se probó que se está lejos del modelo de competencia perfecta, por lo que habría evidencia a favor del ejercicio de poder mercado en el mercado mayorista de leche.

\section{Estimaciones paramétricas.}

Este capítulo estará dedicado a las estimaciones paramétricas. A partir de técnicas econométricas se estimarán los parámetros que permiten identificar el poder de mercado. El programa en el que se realizarán las estimaciones es el Econometric Views 2.0.

Anteriormente, a partir de la maximización de beneficios, se obtuvo una ecuación similar a la que se expone a continuación:

$$
\sum_{i=1}^{n} \kappa_{i} p_{i}-n \frac{\partial r}{\partial X} X-n r=0
$$

\footnotetext{
${ }^{9}$ También apoya las estimaciones paramétricas del capítulo siguiente, ya que también supone que el mercado minorista es competitivo.
} 
Tras un reordenamiento de los términos de la ecuación anterior, podemos derivar la siguiente relación:

$$
r=\frac{1}{n} \sum_{i=1}^{n} \kappa_{i} p_{i}-\frac{\partial r}{\partial X} X
$$

Los $\boldsymbol{\kappa}_{\mathbf{i}}$ 's corresponden a los coeficientes de requerimientos del insumo leche de tal manera que el producto sólo puede ampliarse proporcionalmente a la cantidad de leche. Este es un supuesto razonable para el caso en estudio, ya que el rendimiento es un parámetro estandarizado en la industria. Bajo estas consideraciones, nos encontramos con que el valor de la productividad marginal del insumo leche es constante, dado que habíamos supuesto que el comportamiento minorista era de competencia perfecta.

Como se cuenta con estimaciones alternativas de los $\boldsymbol{\kappa}_{\mathbf{i}}$ 's, construyo una nueva variable que llamo $\mathbf{P}$, definida como

$$
P_{t}=\sum_{i=1}^{n} \kappa_{i, t} p_{i, t}
$$

La ecuación a estimar que corresponde a la pseudo-demanda para las empresas mayoristas es la siguiente:

(8) $r=c+\theta P-(\lambda / \beta) X$,

donde $\boldsymbol{\lambda}$ es el parámetro que va a caracterizar el poder de mercado ${ }^{10}$ y $\boldsymbol{\beta}$ es la pendiente de la oferta. La expectativa sobre $\boldsymbol{\lambda}$ es que esté entre cero y uno. Estos valores extremos caracterizan un mercado competitivo o un monopsonio o cartel perfecto, respectivamente. Valores intermedios corresponderían a alguna solución de un modelo

\footnotetext{
${ }^{10}$ Sobre la justificación de $\lambda$ en la ecuación (7) véase Bresnahan(1982). Ver Lau(1982) para un análisis formal sobre la identificación de $\lambda$.
} 
de oligopsonio. $\boldsymbol{\lambda}$ debe ser interpretado como la conducta promedio de la industria en el período muestral.

El parámetro $\boldsymbol{\theta}$ captará la relación con respecto a todos los precios de los productos finales; por consiguiente se espera que sea positivo. c es la constante de estimación.

Las estimaciones de SAGPyA(1997) de los rendimientos promedio del insumo leche para los distintos productos, para el año 1996 es la siguiente:

$\begin{array}{lc}\text { queso pasta dura } & 7,18 \% \\ \text { queso pasta semidura } & 9,23 \% \\ \text { queso pasta blanda } & 13,00 \% \\ \text { leche en polvo descremada } & 8,00 \% \\ \text { dulce de leche } & 57,14 \% \\ \text { manteca } & 5,68 \% \\ \text { yogur } & 97,09 \% .\end{array}$

Para la leche pasteurizada estimé un valor de $0,96 \%$.

Corresponde ahora analizar el lado de la oferta. El proceso productivo se inicia en el tambo. Los tamberos o productores están atomizados y son tomadores de precio. Al ser la producción de cada unidad productiva insignificante respecto del total, realmente el mercado de leche cruda genera una función de oferta en condiciones de competencia perfecta. Además, no hay clubes de productores que puedan ejercer cierta presión negociadora en la comercialización con las empresas lácteas.

El ciclo normal del animal, a lo largo de su vida útil y desde el momento de su primer parición, es producir leche por 300 días, salir del tambo con siete meses de preñes y, luego de dos meses sin producir leche, parir y repetir el proceso. Es decir, la cantidad de animales en un momento dado del tiempo está predeterminada. Lo que si puede variarse es la proporción entre animales dentro y fuera del tambo en el corto 
plazo $^{11}$. La tasa de interés como variable dentro de la ecuación de la oferta se incluye para captar el costo de oportunidad fuera de la economía, donde el productor podría invertir su dinero en caso de racionalizar el tambo ${ }^{12}$. Se espera a priori un signo negativo sobre el parámetro que mide su significatividad.

Ahora hay que considerar la producción en cada animal. A través de la alimentación es posible modificar la cantidad de leche que se ordeña en cada animal. La relación se da porque la cantidad que injiere se transforma en materia seca y, dentro de cierto rango bastante amplio, la materia seca hace su conversión a leche ${ }^{13}$. El productor, entonces, maximiza en cada momento del tiempo su función de beneficios teniendo en cuenta el precio que recibe, el costo de la suplementación ${ }^{14}$ y las oportunidades inmediatas para llevar su dinero al sector financiero. El suplemento alimenticio más relevante es el concentrado lechero ${ }^{15}$; que es el producto con mayor porcentaje de materia seca. Se espera que el signo de su coeficiente sea negativo. Las restricciones que enfrenta en el corto plazo son las variables que tiene predeterminadas como la cantidad de animales, la calidad de la hacienda, su etapa de gestación promedio y la cantidad de hectáreas sembradas.

Se postula una función de oferta lineal como la siguiente:

(9) $X=\alpha+\alpha_{1} e n e+\alpha_{2} f e b+\ldots+\alpha_{11} n o v+\beta r+\gamma b a l+\delta$ int .

donde $\boldsymbol{\beta}$, como se mencionó, es la pendiente de la oferta. $\boldsymbol{\gamma}$ y $\boldsymbol{\delta}$ captan los efectos sobre bal (precio del alimento balanceado ${ }^{16}$ ) y int (tasa de interés pasiva para plazos fijos en pesos ${ }^{17}$ ) respectivamente. Las $\boldsymbol{\alpha}$ 's captan los efectos de las variables dummies mensuales incluidas de acuerdo a la estacionalidad encontrada en la producción.

\footnotetext{
${ }^{11}$ Es decir, la cantidad de animales que se encuentra efectivamente en la producción.

${ }^{12}$ Sea por descarte anticipado de vacas o cierre del establecimiento. Heien (1977) utiliza la tasa de desempleo para este propósito.

${ }^{13}$ Ver Owen (1983) y Melo y Danelón (1988).

${ }^{14}$ Se refiere a los alimentos fuera del pasto natural.

15 Ver Rearte (1993) sobre la importancia del concentrado en los rodeos argentinos.

${ }^{16}$ Extraído de varios números de la revista Márgenes Agropecuarios.

${ }^{17}$ La fuente es el Banco Central de la República Argentina.
} 
Las ecuaciones a estimar son la (8) y la (9), a las cuales se les añade en forma aditiva un término de error bajo los supuestos adecuados. Nuestro parámetro de interés es el índice de Lerner, que se obtiene luego de la estimación de $(\boldsymbol{\lambda} \boldsymbol{\beta})$ multiplicando por $\mathbf{r}$ y dividiendo por $\mathbf{X}$. También se aplicará el test de Wald a la estimación de $\boldsymbol{\lambda}$, para ver si difiere de cero y de uno.

El método de estimación es el de mínimos cuadrados en tres etapas en forma iterada, que es asintóticamente equivalente a la estimación máximo verosímil ${ }^{18}$. Se realizaron varias estimaciones, cambiando los instrumentos de estimación, verificándose que la estimación del parámetro de poder de mercado fuese robusta a las modificaciones y siempre significativa al $1 \%$.

La estimación más adecuada en términos estadísticos es la presentada en el cuadro $2^{19}$.

Como puede verse, el parámetro $\lambda$ está identificado y su estimación es 0,52. El índice de Wald rechaza tanto la hipótesis nula de que sea igual a cero como de que sea igual a uno con una probabilidad de menos del $1 \%$. De esa manera, aunque no habría poder de mercado extremo como en el caso de un monopsonio, hay evidencia en favor de rechazar la hipótesis de competencia.

El índice de Lerner se obtiene de $(\boldsymbol{\lambda} \boldsymbol{\beta})^{*} \mathbf{X} / \mathbf{r}^{20}$. El valor promedio del índice de Lerner es 0,51. Como podemos ver, si la conducta permanece fija, el índice de Lerner varía con la elasticidad de la oferta.

\footnotetext{
${ }^{18}$ Ver Hausman (1983).

${ }^{19}$ Los meses de octubre, noviembre, diciembre y enero concentran la ordenada al origen en $\alpha$, dado que en todas las estimaciones nunca se pudo rechazar la hipótesis nula del test de significatividad, ni a altos valores como el $10 \%$.

20 * es por "estimado".
} 
CUADRO 2: ESTIMACIÓN DE LA OFERTA Y DEMANDA DE LECHE EN EL MERCADO MAYORISTA

$\begin{array}{ccc}\text { parámetros } & \text { estimación } & \text { desvío estándar } \\ \lambda / \beta & -0,000135 & 0,0000197 \\ \beta & 3841,308 & 887,5203 \\ \theta & 0,020747 & 0,001961 \\ \gamma & -542,4427 & 309,8202 \\ \delta & -3,998367 & -1,961489 \\ c & 0,082071 & 0,010597 \\ \alpha & 238,0736 & 167,5246 \\ \alpha 2 & -183,016 & 27,20207 \\ \alpha 3 & -195,9724 & 29,74005 \\ \alpha 4 & -288,2212 & 35,13156 \\ \alpha 5 & -248,1570 & 39,42853 \\ \alpha 6 & -250,3030 & 40,47847 \\ \alpha 7 & -203,3041 & 37,14333 \\ \alpha 8 & 177,9466 & 32,16651 \\ \alpha 9 & -117,4750 & 26,39667\end{array}$

El $R^{2}$ para la estimación de la ecuación (8) es 0,437195.

El R ${ }^{2}$ para la estimación de la ecuación (9) es 0,405514.

Un detalle teórico respecto a la elasticidad de la oferta surge de una derivación análoga a la relación entre el ingreso marginal y la elasticidad de la demanda para un monopolio. En el caso de un monopsonio la relación sería entre el gasto marginal y la elasticidad de la oferta El resultado predice que la elasticidad de la oferta debería ser siempre menor que uno si el monopsonista (o cartel perfecto) es un maximizador de beneficios. A continuación presentamos algunas conclusiones de las estimaciones de la elasticidad de la oferta. 
El promedio de la elasticidad de la oferta para el período fue de 1,07, que es bastante cercano a uno. Puede argumentarse, entonces, que para algunos meses estará por debajo de 1 , de acuerdo con el caso de monopsonio. Utilizando un índice promedio de estacionalidad mensual, podemos ver que en los meses de octubre, noviembre, diciembre y enero la elasticidad de la oferta es menor a uno, lo que se corresponde con los valores más altos para el índice de Lerner, ceteris paribus el comportamiento de los mayoristas. Es interesante notar que los meses mencionados se corresponden con los picos en la producción. Es consistente, entonces, que en esos meses sea realmente alto el poder de mercado de los mayoristas, dado que los productores no pueden almacenar la leche, que debe ser entregada si o si a la usina o al fabricante.

\section{Conclusiones.}

El análisis de los datos realizado durante los capítulos anteriores permite concluir que el mercado primario de leche en Argentina no funciona de acuerdo al modelo de competencia perfecta. La consecuencia directa es que los productores rurales pierden un porcentaje de la rentabilidad que se genera en la industria y que les correspondería si no se dieran las fallas de mercado.

En primera instancia, los datos no pudieron rechazar la hipótesis de la maximización del beneficio en dos pruebas no paramétricas. Este resultado permite avanzar en la contrastación de la hipótesis de ejercicio de poder de mercado con las pruebas paramétricas y no paramétricas elegidas. Al efectuar dichas pruebas llegamos a la conclusión de que parecería existir un grado intermedio de poder de mercado, sin llegarse al caso de monopsonio o cartel perfecto (aunque la estimación paramétrica sugiere que un comportamiento extremo de monopsonio podría operar en los meses de sobreproducción). Ambas pruebas son consistentes entre si y robustecen los resultados.

Una posible línea de investigación complementaria a la del presente trabajo sería estimar los parámetros de un modelo conjunto de oferta y demanda de leche en los mercados mayorista y minorista, en el cual se introdujera al mismo tiempo a los 
productores rurales, mayoristas, minoristas y consumidores finales (incorporando eventualmente también al sector externo).

Otra línea de investigación que se desprende del presente trabajo es la de incluir la posibilidad de discriminación de precios por parte del mayorista hacia el productor rural, y de este modo separar los problemas que el ejercicio de poder de mercado genera sobre la eficiencia y sobre la distribución del ingreso. 


\section{Referencias bibliográficas}

Bresnahan, Timothy F. (1982), "The Oligopoly Solution Concept is Identified", Economics Letters, 10:87-92.

Hausman, Jerry A. (1983), "Specification and Estimation of Simultaneous Equation Models", en Z. Griliches y M. D. Intriligator, Eds., Handbook of Econometrics, Vol. I, North-Holland Publishing Company.

Heien, Dale (1977), “The Cost of the U.S. Dairy Price Support Program: 1949-74”, The Review of Economics and Statistics, Vol. 59:1-8.

Lau, Lawrence J. (1982), “On Identifying the Degree of Competitiveness from Industry Price and Output Data, 10:93-99.

Lamisovsky, Gustavo (1998), “Concentración y Competencia en la Industria Láctea Argentina”. Tesis de posgrado de Economía de Gobierno, Universidad de San Andrés.

Lerner, A. P. (1934), “The Concept of Monopoly and the Measurement of Monopoly Power, Review of Economics Studies, 11:157-175.

Love, Alan H. y C. Richard Shumway (1994), "Nonparametric Tests for Monopsonistic Market Power Exertion”, American Journal of Agriculture Economics, 76:11561162.

Martin, Stephen, Advanced Industrial Economics, Blackwell Publishers, 1993.

Melo, Oscar, Castillo, Alejandro y José Luis Danelón (1988), Cálculo de Requerimientos Energéticos en Vacas Lecheras, Editorial Hemisferio SUR S.A. 
Noelke, Corinna M y Kellie Curry Raper (1998), "Parametric and Nonparametric Market Power Tests: an empirical investigation", Selected Paper presented at the AAEA Annual Meetings SALT Lake City, UTA Augest 4, 1998.

Owen, John B. (1983), Cattle Feeding, Farming Press Ltd., Ipswich, Great Britain.

Parellada, Gabriel y Ernesto Schilder (1999), “Transformaciones Cíclicas y Estacionales de la Producción Lechera Argentina a partir del Plan de Convertibilidad", Instituto de Economía y Sociología del INTA, Documento de Trabajo No 4, febrero de 1999.

Rearte, Daniel H. (1993), Alimentación y Composición de la Leche en los Sistemas Pastoriles, INTA Balcarce.

Secretaría de Agricultura, Ganadería, Pesca y Alimentación -SAGPyA- (1997), Informe Estadístico de Leche y Productos Lácteos 1996.

Secretaría de Agricultura, Pesca y Alimentación -SAPyA- (1996), Principales Cuencas Lecheras Argentinas.

Varian, Hal R. (1984), "The Nonparametric Approach to Production Analysis", Econometrica, 52, pp. 579-597.

Varian, Hal R.(1990), "Goodness of Fit in Optimizing Models", Journal of Econometrics, 46:125-140.

Varian, Hal R. (1996), "Efficiency in Production and Consumption", en Hal R. Varian, Ed., Computational Economics and Finance, TELOS/Springer-Verlag. Reimpreso en Mathematica in Education and Research, 6(3), 1997. 